\title{
Development of the Microbiota and Associations With Birth Mode, Diet, and Atopic Disorders in a Longitudinal Analysis of Stool Samples, Collected From Infancy Through Early Childhood
}

Citation for published version (APA):

Galazzo, G., van Best, N., Bervoets, L., Dapaah, I. O., Savelkoul, P. H., Hornef, M. W., Lau, S., Hamelmann, E., Penders, J., GI-MDH consortium, \& Mommers, M. (2020). Development of the Microbiota and Associations With Birth Mode, Diet, and Atopic Disorders in a Longitudinal Analysis of Stool Samples, Collected From Infancy Through Early Childhood. Gastroenterology, 158(6), 1584-1596.

https://doi.org/10.1053/j.gastro.2020.01.024

Document status and date:

Published: 01/05/2020

DOI:

10.1053/j.gastro.2020.01.024

Document Version:

Publisher's PDF, also known as Version of record

Document license:

Taverne

Please check the document version of this publication:

- A submitted manuscript is the version of the article upon submission and before peer-review. There can be important differences between the submitted version and the official published version of record. People interested in the research are advised to contact the author for the final version of the publication, or visit the $\mathrm{DOI}$ to the publisher's website.

- The final author version and the galley proof are versions of the publication after peer review.

- The final published version features the final layout of the paper including the volume, issue and page numbers.

Link to publication

\footnotetext{
General rights rights.

- You may freely distribute the URL identifying the publication in the public portal. please follow below link for the End User Agreement:

www.umlib.nl/taverne-license

Take down policy

If you believe that this document breaches copyright please contact us at:

repository@maastrichtuniversity.nl

providing details and we will investigate your claim.
}

Copyright and moral rights for the publications made accessible in the public portal are retained by the authors and/or other copyright owners and it is a condition of accessing publications that users recognise and abide by the legal requirements associated with these

- Users may download and print one copy of any publication from the public portal for the purpose of private study or research.

- You may not further distribute the material or use it for any profit-making activity or commercial gain

If the publication is distributed under the terms of Article $25 \mathrm{fa}$ of the Dutch Copyright Act, indicated by the "Taverne" license above,

Download date: 26 Apr. 2023 


\title{
Development of the Microbiota and Associations With Birth Mode, Diet, and Atopic Disorders in a Longitudinal Analysis of Stool Samples, Collected From Infancy Through Early Childhood
}

\author{
Gianluca Galazzo, ${ }^{1,2, *}$ Niels van Best, ${ }^{1,3,4, *}$ Liene Bervoets, ${ }^{1,4}$ Isaac Oteng Dapaah, \\ Paul H. Savelkoul, ${ }^{1,2,5}$ Mathias W. Hornef, ${ }^{3}$ the GI-MDH consortium, Susanne Lau, ${ }^{6, \S}$ \\ Eckard Hamelmann, ${ }^{7, \S}$ and John Penders ${ }^{1,2,4, \S}$
}

${ }^{1}$ School of Nutrition and Translational Research in Metabolism (NUTRIM), Department of Medical Microbiology, Maastricht University Medical Centre, Maastricht, the Netherlands; ${ }^{2}$ School for Public Health and Primary Care (Caphri), Department of Medical Microbiology, Maastricht University Medical Centre, Maastricht, the Netherlands; ${ }^{3}$ Institute of Medical Microbiology, RWTH University Hospital Aachen, RWTH University, Aachen, Germany; ${ }^{4}$ in Vivo Planetary Health: an affiliate of the World Universities Network (WUN), West New York, New Jersey; ${ }^{5}$ Department of Medical Microbiology and Infection Control, Amsterdam UMC, location VUmc, Amsterdam, The Netherlands; ${ }^{6}$ Department of Pediatric Pulmonology, Immunology and Intensive Care Medicine, Charité Universitätsmedizin Berlin, Germany; and ${ }^{7}$ Children's Center Bethel, Protestant Hospital Bethel, University of Bielefeld, Germany

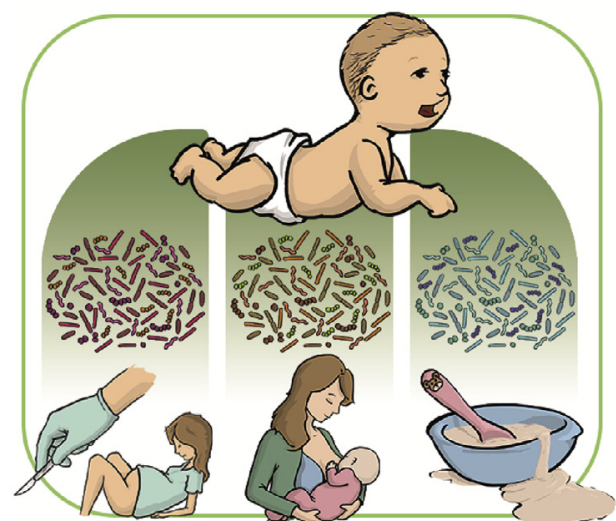

Determinants of microbiome development

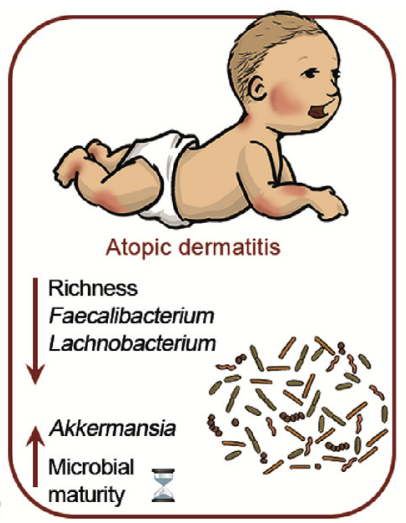

First 100 days of life

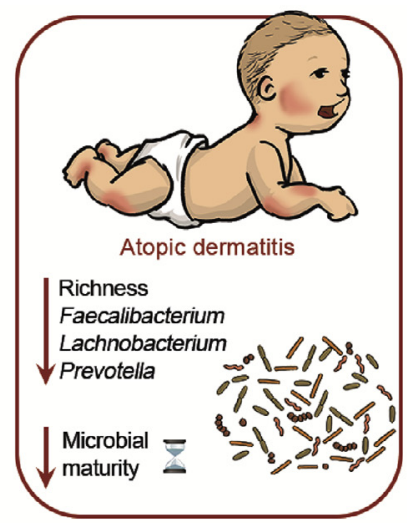

100-200 days of life
BACKGROUND \& AIMS: Establishment of the gastrointestinal microbiota during infancy affects immune system development and oral tolerance induction. Perturbations in the microbiome during this period can contribute to development of immunemediated diseases. We monitored microbiota maturation and associations with subsequent development of allergies in infants and children. METHODS: We collected 1453 stool samples, at 5, 13,21 , and 31 weeks postpartum (infants), and once at school age (6-11 years), from 440 children ( $49.3 \%$ girls, $24.8 \%$ born by cesarean delivery; all children except for 6 were breastfed for varying durations; median 40 weeks; interquartile range, 30-53 weeks). Microbiota were analyzed by amplicon sequencing. Children were followed through 3 years of age for development of atopic dermatitis; data on allergic sensitization and asthma were collected when children were school age. RESULTS: Diversity of fecal microbiota, assessed by Shannon index, did not differ significantly among children from 5 through 13 weeks after birth, but thereafter gradually increased to 21 and 31 weeks. Most bacteria within the Bacteroidetes and Proteobacteria phyla were already present at 5 weeks after birth, whereas many bacteria of the Firmicutes phylum were acquired at later times in infancy. At school age, many new Actinobacteria, Firmicutes, and Bacteroidetes bacterial taxa emerged. The largest increase in microbial diversity occurred after 31 weeks. Vaginal, compared with cesarean delivery, was most strongly associated with an enrichment of Bacteroides species at 5 weeks through 31 weeks. From 13 weeks onward, diet became the most important determinant of microbiota composition; cessation of breastfeeding, rather than solid food introduction, was associated with changes. For example, Bifidobacteria, staphylococci, and streptococci significantly decreased on cessation of breastfeeding, whereas bacteria within the Lachnospiraceae family (Pseudobutyrivibrio, Lachnobacterium, Roseburia, and Blautia) increased. When we adjusted for confounding factors, we found fecal microbiota composition to be associated with development of atopic dermatitis, allergic sensitization, and asthma. Members of the Lachnospiraceae family, as well as the genera Faecalibacterium and Dialister, were associated with a reduced risk of atopy. 
CONCLUSIONS: In a longitudinal study of fecal microbiota of children from 5 weeks through 6 to 11 years, we tracked changes in diversity and composition associated with the development of allergies and asthma.

Keywords: Eczema; Microbial Diversity; Microbial Age; Specific IgE.

olonization of the intestinal tract during the neonatal period is of crucial importance for the maturation of the mucosal immune system and the induction of oral tolerance. $^{1-3}$ Animal studies have provided compelling evidence to support a causal role of the intestinal microbiota and its metabolites, especially in early life, in the etiology of allergic diseases. ${ }^{3-6}$

Numerous epidemiological studies ${ }^{7-12}$ also suggest that the infant intestinal microbiota plays an important role in the manifestation of allergic diseases and asthma, although actual results vary considerably between studies. Approximately half of the studies that examined intestinal microbial diversity in infancy and childhood reported a lower diversity among children with (subsequent) allergies, whereas the remaining studies found no evidence for such an association. ${ }^{13}$ Moreover, despite that many specific microbial taxa have been linked to allergies and asthma, it remains unclear which bacterial taxa prevent or promote disease onset. ${ }^{14}$

Lack of early stool sampling and different ages of stool sample collection, different microbiological profiling methods, and an inadequate control for potential confounders have been suggested to contribute to the heterogeneity between study results. ${ }^{9,13}$ In addition, crosssectional studies are prone to reverse causality, that is, changes in the microbiota composition as a result of the disease manifestation, and only very few studies have sufficient clinical follow-up to link infant microbiota maturation to the subsequent development of asthma. ${ }^{11}$

Initial microbial colonization starts on rupture of the amniotic membranes and subsequent passage through the birth canal when the infant is seeded by maternal microbial strains, a process that is impeded in case of a cesarean delivery. ${ }^{15,16}$ Subsequently, microbial populations evolve as the diet changes and the host develops. Given the highly dynamic and complex process of microbial assembly, succession, and maturation, repeated sampling is important to allow analysis of the overall development of the indigenous infant microbial ecology. ${ }^{17}$ Moreover, many of the antenatal and postnatal factors that influence microbial community assembly during infancy, such as birth mode and the presence of older siblings and furry pets in the household, have also been associated with the development of allergic diseases and asthma, $9,13,18,19$ highlighting the importance to account for potential confounding factors.

In the present study, we aimed to monitor microbial assembly, succession, and maturation during the first year of life and identify hereditary, perinatal, environmental, lifestyle, and dietary factors that drive microbiota development. Through the application of various multivariable

\section{WHAT YOU NEED TO KNOW \\ BACKGROUND AND CONTEXT}

Establishment of the gastrointestinal microbiota during infancy affects immune system development and oral tolerance induction.

\section{NEW FINDINGS}

In a longitudinal study of fecal microbiota of children from 5 weeks through 6-11 years, we tracked changes in microbiota diversity and composition associated with birth mode, diet, and development of allergies and asthma.

\section{LIMITATIONS}

This was a post-hoc analysis of participants in an interventional study. Studies are needed to determine whether these microbes cause or are a result of the observed associations

\section{IMPACT}

Based on analyses of fecal samples, the intestinal microbiota increases in diversity from age 13 weeks through 6-11 years. Changes associate with vaginal vs cesarean section delivery, the end of breastfeeding, and development of allergies and asthma.

longitudinal models, including joint modeling, we next examined the dynamics in microbial diversity, composition, and community structure in association with the subsequent risk of developing atopic dermatitis and asthma until school age.

Our findings indicate that alterations in microbial diversity and composition precede the onset of allergic manifestations, while emphasizing the importance of possible confounders.

\section{Methods}

\section{Design and Clinical Outcome Measurements}

Originally this study was designed as a randomized placebocontrolled clinical trial to examine the impact of a bacterial lysate, containing heat-killed Escherichia coli and Enterococcus faecalis, on the primary prevention of atopic dermatitis (AD) (registration no. ISRCTN60475069, ISRCTN registry). ${ }^{20}$ However, we did not find any evidence that the intervention affected the microbiota composition and therefore pooled both treatment arms in the downstream statistical analyses.

Infants were clinically examined by a pediatrician on signs of $\mathrm{AD}$ at the ages of 1,21 , and 31 weeks and again at 1,2 , and 3 years of age, as described previously. ${ }^{20}$

* Authors share co-first authorship; ${ }^{\S}$ Authors share co-senior authorship.

Abbreviations used in this paper: AD, atopic dermatitits; COPSAC Copenhagen Prospective Study on Asthma in Childhood; DMM, Dirichlet Multinomial Mixture; FDR, false discovery rate; GI, gastrointestinal; Ig,

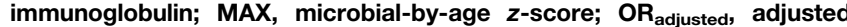
odds ratio; OUT, operational taxonomic unit; rRNA, ribosomal RNA.

\section{Most current article}

(C) 2020 by the AGA Institute $0016-5085 / \$ 36.00$

https://doi.org/10.1053/j.gastro.2020.01.024 
The school-age follow-up of the study population (at 6-11 years) took place in 2013, including clinical examination, lung function testing, skin prick tests, and serum analyses of specific immunoglobulin (Ig)E to the most common aeroallergens (house dust mite, dog, cat, mold [Alternaria, Cladosporium], birch, and grass pollen). Children were classified as having current asthma in case of a doctor's diagnosis in combination of any indicative symptoms in the past 12 months (wheezing, shortness of breath, nocturnal awakening due to shortness of breath and/or wheezing). Allergic sensitization was assessed by Skin Prick Test and serum sensitization for the previously mentioned allergens.

The study and follow-up were approved by the hospital's local review board Charité Ethics Committee in 2002 and 2012. Parents and participants gave written informed consent.

\section{Microbial Profiling of Fecal Samples}

Fecal DNA was isolated by a combination of bead beating and column-based purification as described in detail previously. $^{21}$

Barcoded universal primers adapted from Bartram and collegues $^{22}$ were used to amplify the variable 3 region of the $16 \mathrm{~S}$ ribosomal RNA (rRNA) gene. Amplicons were sequenced using the Illumina (San Diego, CA) MiSeq platform using
GUniFrac $1.1^{26}$ was used to compute the unweighted UniFrac metric.

Ordination of samples based on the unweighted UniFrac dissimilarity was visualized using principal coordinate analysis using the capscale function of the vegan package.

The Friedman test was used to test for significant differences in Chao1, Shannon, and loadings scores on the principal coordinates between all time points, followed by Dunn's test for post hoc pairwise comparisons between individual time points. The $P$ values were finally false discovery rate (FDR) adjusted for multiple comparisons.

To track the dynamics of individual OTUs within the 4 main bacterial phyla, we created Sankey plots using Sankeymatic. For readability of the Sankey plots, the OTU table was further filtered to OTUs that were present in $\geq 10 \%$ of the samples during 1 or more time points.

We next investigated the maturity of the GI microbiota by computing the microbial-by-age $z$-score (MAZ) of the sample as described previously. ${ }^{27}$ Shortly, we started feeding a random forest with a training set made of the microbial community composition of healthy children after filtering out all OTUs with a prevalence less than $5 \%$. Once the model was trained, we used it to predict the age of all the samples. Finally, the $z$-score was computed using the following formula:

$M A Z=\frac{\text { Microbial age }- \text { median of microbial age of healthy children of same chronologic age }}{(\text { standard deviation of microbial age of healthy children of the same chronologic age })}$

$2 \times 250$ paired-end reads. The resulting sequencing data were processed using the short-read library $16 \mathrm{~S}$ rRNA gene sequencing pipeline $(\mathrm{sl} 1 \mathrm{p})^{23}$ (for description, see the supplementary methods).

This resulted in a total of 93,475,612 reads from 1468 samples that were clustered into 7961 operational taxonomic units (OTUs). Removal of OTUs that were observed in only a single sample and discarding OTUs with a fraction of the total number of sequences below 0.001, retained most sequences $(92,997,277)$ while significantly reducing the number of OTUs to 873. Finally, we eliminated 15 samples with a low coverage $\left(<15,000\right.$ reads) and normalized the data using Rhea. ${ }^{24}$ To not discard informative information, normalization in Rhea is performed by dividing OTU counts per sample for their total count (sample depth) and then multiplying the obtained relative abundance for the lowest sample depth $(15,540$ reads).

\section{Statistical Analysis}

All the statistical analyses were performed 2-sided using R, version 3.4.3.

Gastrointestinal (GI) microbiota richness and maturation. The Chao1 index, as measure for the estimated microbial richness, and the Shannon index, as microbial diversity metric, were computed using the $\mathrm{R}$ package vegan 2.5.3. ${ }^{25}$

To compare the microbial community structure of samples, we used the unweighted UniFrac, which incorporates phylogenetic distances between observed organisms. The $\mathrm{R}$ package
Dirichlet Multinomial Mixture (DMM) clustering, an unsupervised clustering method that uses Laplace approximation to identify groups of communities (enterotypes) with similar composition, was performed as previously described. ${ }^{28} \mathrm{We}$ then analyzed the transition of infants through these DMM clusters with age. ${ }^{29}$

Analysis of factors shaping the GI microbiota. We examined which hereditary, perinatal, environmental, lifestyle, and dietary factors were associated with the establishment of the microbiome during infancy (see supplementary methods for detailed description).

To examine which of these factors were associated with the DMM clusters at baseline and/or with the transition of DMM clusters between the ages of 5 and 31 weeks, multinomial logistic regression analyses were used. Only factors that were significantly associated with the (transition of) DMM clusters in the univariable analyses were included in the final multivariable model.

We next used multivariate association with linear models (MaAsLin) ${ }^{30}$ to examine the association between these factors with and individual microbial taxa and multivariable linear regression models to examine the association with the microbial diversity and maturity.

The effect size and significance of each of the covariates on the microbial community structure was determined using the envfit function in vegan. ${ }^{25}$ Ordination was performed using the principal coordinate analysis based on unweighted UniFrac metric obtained as described previously. The significance value was determined based on 999 permutations. All $P$ values 
derived from envfit were adjusted for multiple comparisons using FDR adjustment (Benjamini-Hochberg procedure).

To understand which of the covariates had the strongest impact on the overall microbial community structure, we performed a permutational analysis of variance based on unweighted UniFrac. Only covariates that were statistically significant in the envfit analyses were included in the permutational analysis of variance.

Analysis on association between microbiota and allergic manifestations. To examine how the longitudinal variation of the microbial diversity (Shannon index) and maturity (MAZ) of the GI microbiota affects the time to development of $\mathrm{AD}$, we applied a joint model $^{31}$ using the JM function of the JMpackage ${ }^{32}$ (for details, see the supplementary methods).

To examine the impact of microbial diversity and maturity on asthma and allergic sensitization at school age, a generalized linear model was built using Ime41.1.19. The same covariates as included in the JM were incorporated as potential confounding factors. Because both asthma and sensitization were binary outcomes, a binomial distribution was chosen for the generalized linear model.

To identify if specific bacterial genera were differentially abundant in children with and without allergic manifestations, we used the MetaLonDa package. ${ }^{33}$ To ensure meaningful $P$ values, we performed 999 permutations. To select only the significant associations, we chose a threshold of .05 for the $P$ values after FDR adjustment.

\section{Results}

\section{Study Population Characteristics}

The study, initially designed as a randomized, placebocontrolled trial on the primary prevention of AD by an orally applied lysate of heat-killed Escherichia coli and Enterococcus faecalis, consisted of healthy newborns ( $\mathrm{n}=$ 606) with a single or double heredity for atopy. ${ }^{20}$ During the first 3 years of life, children were deeply phenotyped by physical examination and the collection of detailed questionnaires at 7 clinical visits. At school age, children were contacted again to determine the establishment of asthma and allergic sensitization.

For the present study, only children with at least 3 fecal samples collected during the first year and/or feces collected at school age were included $(\mathrm{n}=440)$. Of these children, 217 (49.3\%) were girls, 187 (42.5\%) had older siblings, 109 $(24.8 \%)$ were born by cesarean delivery, and 29 (6.6\%) were reportedly treated with antibiotics in the first 31 weeks of life. All except 6 children received breastfeeding, although the duration of breastfeeding varied considerably, with a median duration of 40 weeks (interquartile range 30-53). Solid food was introduced at a median age of 25 weeks (interquartile range 22-27) (Supplementary Table S1).

\section{Development of the Microbiota Between Early Infancy and School Age}

We first examined the compositional changes in the microbiota during infancy and compared this to the schoolage microbiota composition. Samples collected at the age of $5(\mathrm{n}=306), 13(\mathrm{n}=287), 21(\mathrm{n}=268)$, and $31(\mathrm{n}=307)$ weeks postpartum and again at school age $(n=300)$ were profiled by amplicon sequencing of the 16S rRNA hypervariable V3 gene region. On quality filtering and removal of samples with low sequencing depth $(\mathrm{n}=15), 1453$ samples with a median sequencing depth of 62,420 reads/sample (range 15,540-168,848) were retained for downstream analysis and clustered into 873 OTUs.

Microbial diversity, assessed by the Shannon index, was not significantly different between ages 5 and 13 weeks, but thereafter gradually increased from 13 to 21 and 31 weeks after birth (Figure $1 A$ ). The largest increase in microbial diversity occurred after the age of 31 weeks, as indicated by the steep increase in the Shannon index at school age $(P=7.99 \times$ $\left.10^{-28}\right)$. Similar findings were observed for the microbial richness as assessed by the Chao1 (Supplementary Table 2).

Principal coordinate analyses indicated that the microbial community structure as assessed by the unweighted UniFrac metric also gradually shifted during infancy, with the most prominent shift between the ages of 21 and 31 weeks (Figure $1 B, P=1.58 \times 10^{-27}$, Supplementary Table 3). The school-age samples, however, clustered separately and showed less interindividual variation as compared with the infant samples.

Tracking individual OTUs based on their presence or absence revealed different dynamics within the dominant phyla (Supplementary Figure 1). Most OTUs within the phyla of Actinobacteria, Bacteroidetes, and Proteobacteria found during infancy were already present at 5 weeks after birth, whereas almost half of the OTUs within the phylum of Firmicutes were acquired only at later infant time points. At school age, many new Actinobacteria, Firmicutes, and Bacteroidetes OTUs emerged on top of the OTUs already present during infancy. In contrast, only few new OTUs emerged within the phylum Proteobacteria at school age, whereas a significant portion of the infant OTUs were lost thereafter.

We next examined the bacterial genera that contribute most to the temporal dynamics in microbial diversity and community structure. Toward school age, the prevalence in many of the genera within the phylum of Proteobacteria dramatically decreased, whereas the prevalence of genera within the phylum of Firmicutes, and in particular within the Lachnospiraceae and Ruminococcaceae families strongly increased (Figure 1C). Moreover, with the exception of Bifidobacterium, the relative abundance of all of the major bacterial genera changed significantly (Friedman test, all $P$ values <.001, Supplementary Table 4) throughout infancy and childhood (Supplementary Figure $2 A$ and $B$ ). Escherichia was the most abundant genus at 5 weeks of age followed by Bifidobacterium and Streptococcus. Escherichia still remained the most abundant genus at 31 weeks of age but was now followed by Bacteroides and Veillonella. At school age, the most abundant genera were Blautia, Faecalibacterium, and Ruminococcus.

\section{The Length of Breastfeeding Represents the Main Driver of the Infant's Microbiota Composition}

To identify covariates associated with the microbiota dynamics during infancy, we continued our analyses 

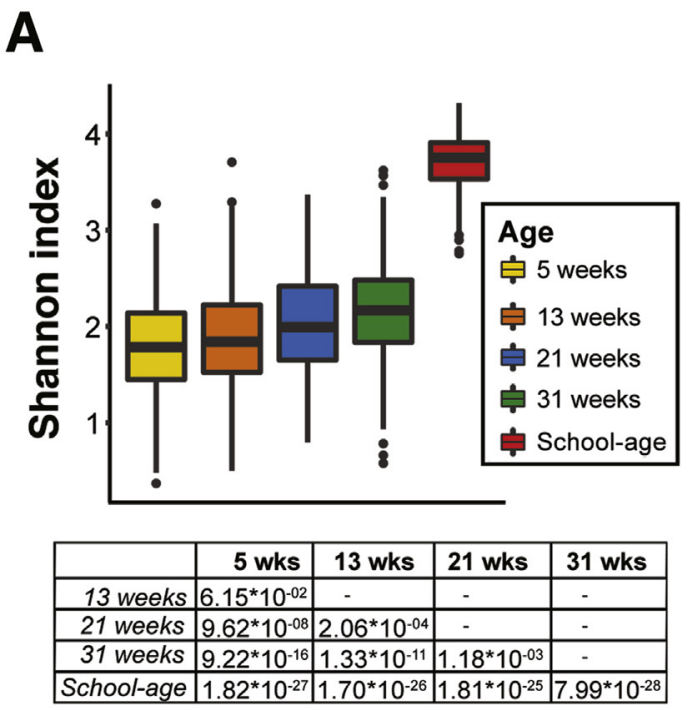
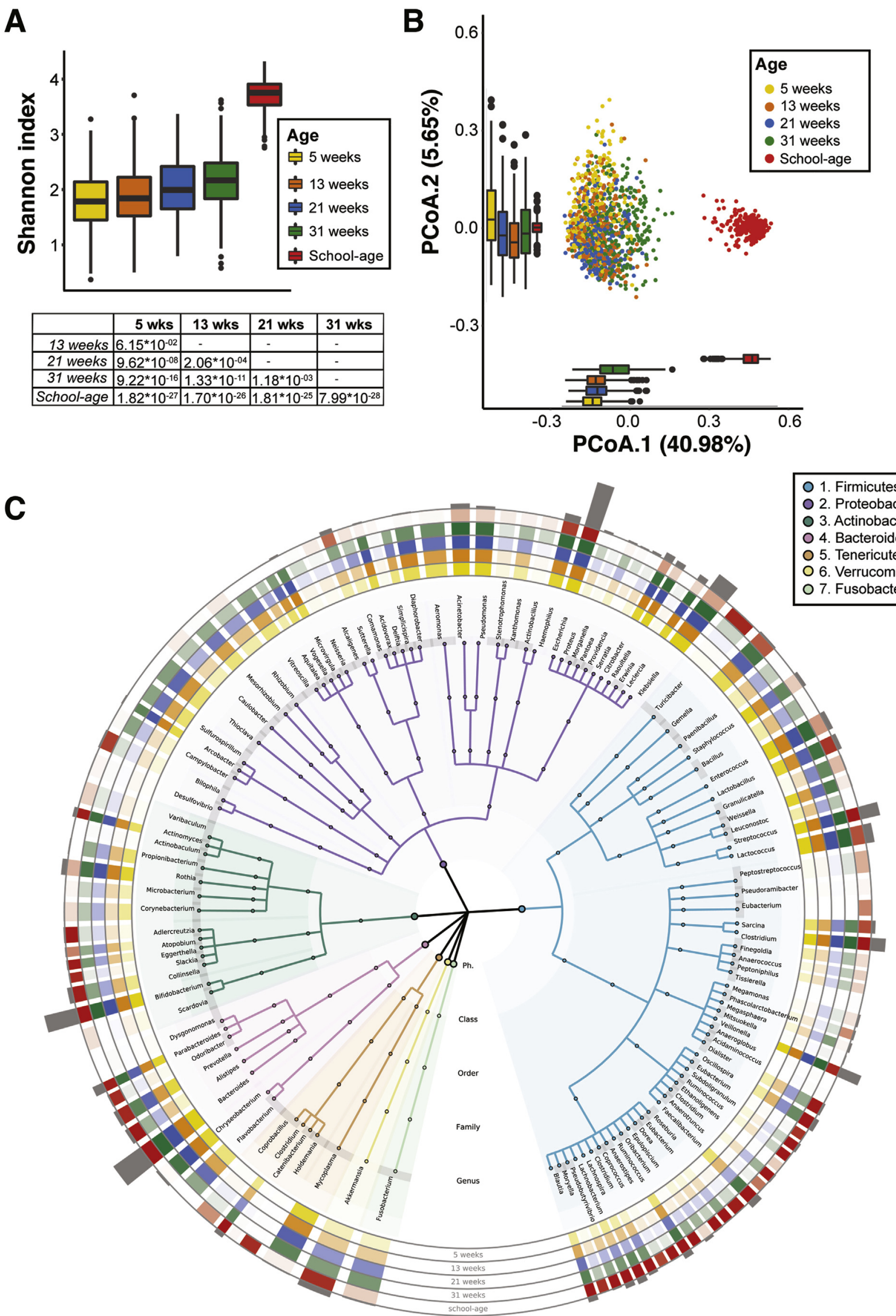
focusing on the infant samples. DMM modeling on OTU-level data formed 6 clusters (based on lowest Laplace approximation) (Figure $2 A$ and $B$ ).

To illustrate the progression of samples through each DMM cluster with age, we applied a transition model as described previously. ${ }^{29}$

Clusters 1 and 3 were the most dominant at the age of 5 weeks and thereafter transitions were chaotic, consistent with the previously identified developmental phase of the microbiome during the first 14 months of life. ${ }^{29}$ Although cluster 1 remained dominant until the age of 31 weeks, cluster 3 gradually disappeared in favor of clusters 4 and 5 (Figure 2C). Multinomial logistic regression analyses indicated that the initial microbiota cluster at 5 weeks of age was mainly determined by birth mode. The chance that a newborn's microbiota belonged to cluster 3 was strongly increased among infants born by cesarean delivery (Supplementary Table 5). This cluster was remarkably different with respect to the abundance of several of the driving OTUs. In particular, a Klebsiella OTU exhibited a high abundance at the expense of an Escherichia OTU that dominated many of the other clusters. In addition, Citrobacter, Leclercia, and Raoultella OTUs were characteristic for cluster 3 (Figure 2B).

Analysis of the most common transition trajectories revealed that for children starting in cluster 1 as well as for children starting in cluster 3 , transition toward clusters 4 and 5 significantly increased when breastfeeding was ceased (Supplementary Table 5).

These results were further supported by the overall bacterial profiles throughout infancy. At the age of 5 weeks, the largest amount of variance was explained by birth mode (Figure $3 A$ ). At the genus level, vaginal as compared with cesarean delivery was most strongly associated with an enrichment of Bacteroides spp at 5 weeks and until the age of 31 weeks (Supplementary Table 6).

At the ages of 13, 21, and 31 weeks, breastfeeding explained by far the greatest variance in bacterial community profiles (Figure $3 B-D$ ). Permutational multivariate analyses of variance confirmed that the duration of breastfeeding had a stronger impact than the introduction of solid foods (Figure $3 E$, Supplementary Table 7). Bifidobacteria, staphylococci, and streptococci, among others, significantly decreased on cessation of breastfeeding, whereas many bacteria within the Lachnospiraceae family (eg, Pseudobutyrivibrio, Lachnobacterium, Roseburia, Blautia) increased (Supplementary Table 6).

A longer duration of breastfeeding was also associated with a lower microbial diversity (Supplementary Table 8), as well as with a lower MAZ (Supplementary Table 9). The MAZ is calculated by training a machine-learning algorithm on the microbiota composition of a dataset with known biological age, thereafter the age of samples is predicted based on its microbiota composition. A lower MAZ is thus indicative for a delayed microbial maturation.

Furthermore, the exposure to older siblings was associated with an increase in several genera within the phylum of Actinobacteria (Bifidobacterium and Corynebacterium at 5 weeks and Eggerthella at 21 weeks, Supplementary Table 6) and a higher microbial diversity at 31 weeks of age (Supplementary Table 9). Finally, besides dietary factors, the microbial community structure was most strongly associated with the presence of $\mathrm{AD}$ at time of sample collection.

\section{Alterations in Microbial Composition, Diversity, and Maturity Precede Manifestations of Atopy}

To further investigate whether differences in microbiota development precede the onset of atopic disease, we applied several longitudinal analyses while controlling for potential confounding factors by adjusting for other covariates.

We first applied multivariate joint models on the microbial diversity and maturity in association with AD. Joint models have become increasingly popular as a statistical framework to concurrently analyze longitudinal data (eg, biomarker evolution) and survival data (eg, time to disease onset). ${ }^{31}$ They have, to our knowledge, not been applied in the microbiome research field so far. While accounting for known risk factors for $\mathrm{AD}$, we found that the temporal pattern of microbial diversity was independently and inversely associated with $\mathrm{AD}$ (hazard ratio $0.21 ; P=1.15 \times$ $10^{-4}$, Figure $4 A$ and $B$, Supplementary Table 10 ), indicating that a lower microbial diversity throughout infancy is associated with an increased risk of AD. For the temporal pattern of microbial maturity, expressed as microbial age $z$ scores, we found a statistically significant positive association with $\mathrm{AD}$ (hazard ratio 1.14; $P=1.94 \times 10^{-5}$; Figure $4 C$ and $D$, Supplementary Table 11).

Next, we used the recently introduced metagenomics longitudinal differential abundance (MetaLonDa) method ${ }^{33}$ to identify time intervals of differentially abundant bacterial genera between infants who did or did not develop AD.

Among children who did not develop AD during followup, the relative abundance of Atopobium (days 25.6 to 79.4, FDR $_{\text {adjusted }} P=7.65 \times 10^{-3}$ ), Corynebacterium (days 126.1 to $151.2, \mathrm{FDR}_{\text {adjusted }} P=9.68 \times 10^{-3}$ ), both members of the phylum Actinobacteria, and Prevotella (days 104.6 to 133.3,

Figure 1. Microbiota maturation throughout infancy and childhood $(n=1453$ stool samples from 440 children). (A) Microbial diversity (Shannon index) gradually increased throughout infancy and has markedly risen at school age $\left(P=7.72 \times 10^{-53}\right.$, Friedman, $P$ values for post hoc analyses using Dunn's test are depicted in the table). (B) Principal coordinate analysis (PCoA) based on unweighted UniFrac dissimilarity indicates a gradual shift in microbial community structure along PC1 during infancy and a completely distinct structure at school age $\left(P=6.0 \times 10^{-51}\right.$, Friedman, $P$ values for post hoc analyses using Dunn's test are depicted in Supplementary Table 3). (C) Cladogram depicting the bacterial genera detected in the children's fecal microbiota. Background and branch colors reflect the different phyla. The height of the outer ring reflects the average relative abundance of a genus across all infant time points, whereas the color density of the 5 inner rings reflects the prevalence of the genus at the individual time points (with opaque color indicating a prevalence of $100 \%$ and fully transparent indicating a prevalence of $0 \%$ ). 
A

PC2

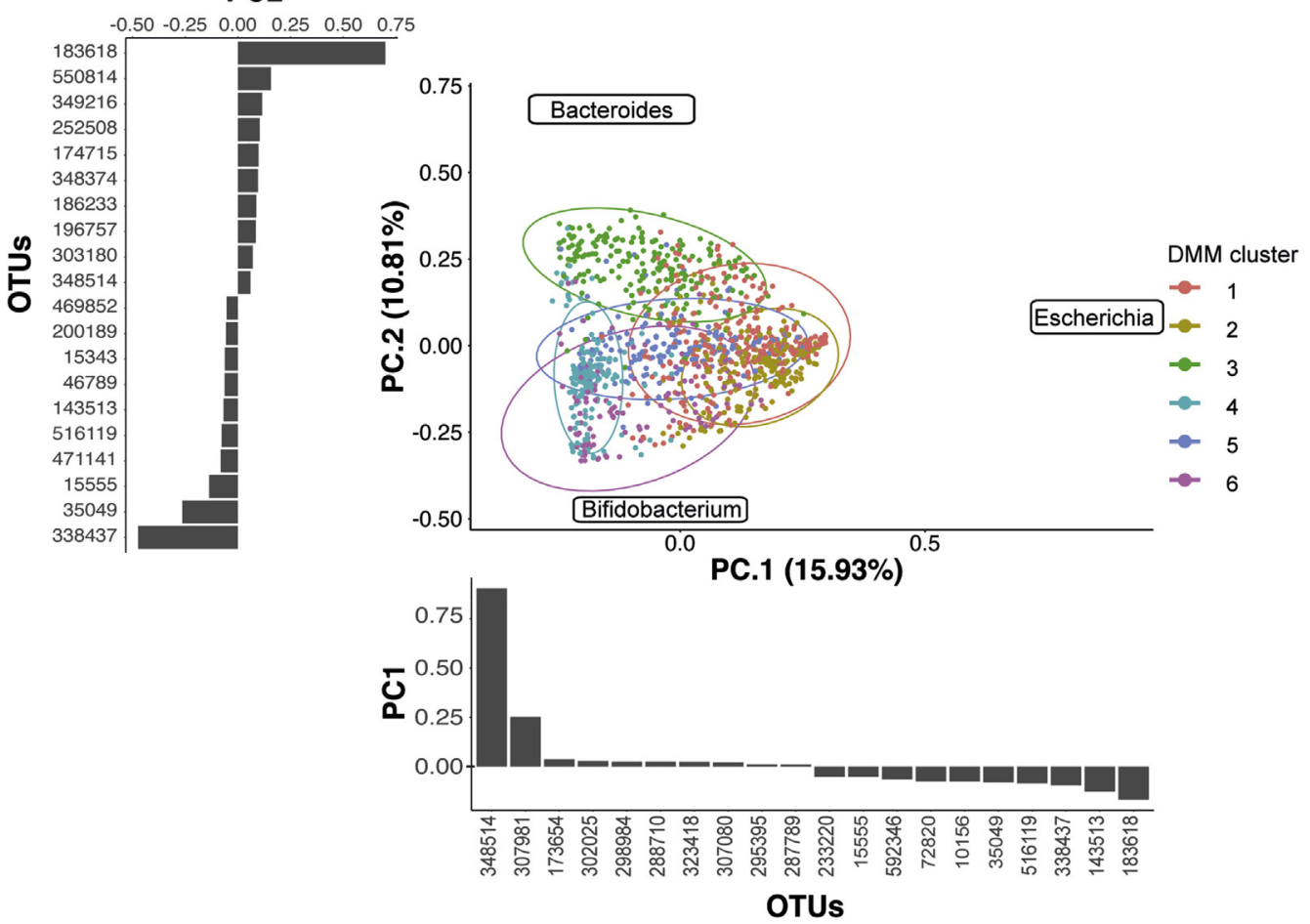

B
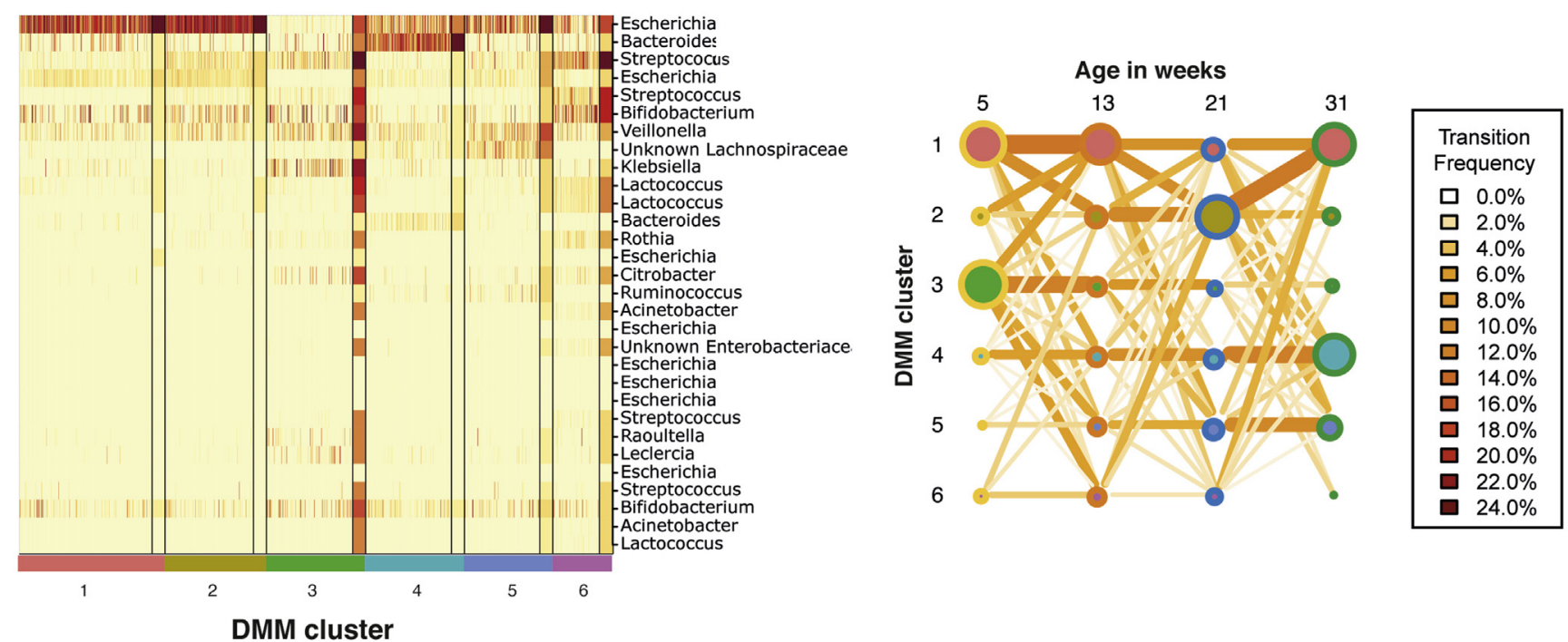

Figure 2. Community typing by DMMs of infant samples revealed 6 clusters ( $\mathrm{n}=1154$ stool samples from 312 children). (A) Heat map showing the relative abundance of the 30 most important/dominant OTUs per DMM cluster. (B) Principal component analysis on OTU-level data with samples colored according to DMM cluster. Ellipses indicate the $95 \%$ confidence interval. Vertical and horizontal bar charts depict OTUs with the highest loadings on PC1 and PC2, respectively. The OTUs with the highest positive and negative loadings on PC1 and PC2 are plotted in the PCA. (C) Transition model showing the progression of samples through the $6 \mathrm{DMM}$ clusters from one sampling time point to the next time point.

FDR $_{\text {adjusted }} P<.001$ ) were temporarily enriched when compared with children who developed AD. Most pronounced were, however, the associations of Lachnobacterium and Faecalibacterium, which were significantly enriched during the entire period of fecal sampling among children who remained free from AD (Figure $4 E-G$, Supplementary Table 12).

We next examined whether the infant microbiota composition was also associated with allergic manifestations at school age, including allergic sensitization and 
asthma. Blood samples for the determination of allergic sensitization at school age were available for 292 of the 440 children included in the present study. Like for $A D$, we found a higher diversity of the infant microbiota to be associated with decreased risk of allergic sensitization at school age (Shannon index at 31 weeks adjusted odds ratio [OR $\left.{ }_{\text {adjusted }}\right] 0.19 ; P=7.33 \times 10^{-3}$, Supplementary Figure $3 A$, Supplementary Table 13). A higher microbial maturity very early in life was associated with an increased risk of allergic sensitization (MAZ at 5 weeks $\mathrm{OR}_{\text {adjusted }} 1.46, P=5.01 \times$ $10^{-3}$, Supplementary Figure $3 B$, Supplementary Table 14), again in line with findings for AD. We could not, however, identify individual bacterial genera with differential abundance over a significant period of time between children who did or did not develop allergic sensitization.

A clear association between microbial diversity and asthma could not be detected. Yet, in line with allergic sensitization and AD, a higher microbial maturity at the age of 5 weeks was also associated with an increased risk for asthma (MAZ at 5 weeks $\mathrm{OR}_{\text {adjusted }} 1.43 ; P=7.78 \times 10^{-3}$, Supplementary Figure $3 C$ and $D$, Supplementary Tables 15 and 16). Multiple bacterial genera were differentially abundant over time in children who did or did not develop asthma. The genera that were differentially abundant across the entire period during which the microbiota composition was monitored included Lachnobacterium, Lachnospira (both members of the Lachnospiraceae family), and Dialister (Veillonellaceae), which were all significantly enriched in healthy as compared with asthmatic children (Supplementary Figure $3 E-G$, Supplementary Table 17).

\section{Discussion}

This study aimed to longitudinally analyze the process of GI microbial community assembly, succession, and maturation throughout the most critical time window of immune development, and linked microbiota maturation during this time to the development of clinical signs of allergic disease, while carefully controlling for potential confounding factors.

Our results indicate a dynamic microbiota during infancy that is far from completely matured at 31 weeks of age. In early infancy, the microbial composition was most strongly affected by birth mode, whereas from 13 weeks onward diet became the most important factor. Our data support previous reports, showing that Bacteroides are most strongly affected by birth mode. ${ }^{29,34-36}$ The difference in microbial community structure and lower abundance of Bacteroides in cesarean delivery as compared with vaginal-delivered infants persisted up to the age of 31 weeks and withstood mutual adjustment for other determinants, including breastfeeding. This suggests that the impact of cesarean delivery could not be compensated by breastfeeding. Given the increased risk of future diseases, including allergies and asthma, $^{37,38}$ among children born by cesarean delivery, more research is warranted to elucidate the need for and efficacy of restoring the natural microbial colonization process on cesarean delivery.

We furthermore showed that cessation of breastfeeding was more strongly associated with microbial composition and maturity than solid food introduction. In line with previous studies, ${ }^{11,35,39}$ these results suggest that the introduction of solid food does not appear to result in a profound shift in microbial community structure as long as breastfeeding is continued. Only when breastfeeding is ceased, maturation of the microbiota is accelerated with a decrease in degraders of human milk oligosaccharides and an increase in microbial diversity and compositional changes toward bacterial genera specialized in degrading complex dietary carbohydrates. The generally observed lower microbial diversity in infants during breastfeeding ${ }^{40}$ seems at first contradictory to the concept that a "healthy" and resilient microbiome is highly diverse. ${ }^{41}$ However, in line with most prospective studies, ${ }^{42}$ we did not find a direct association between breastfeeding duration and the risk of $\mathrm{AD}$. The fact that breastfeeding reduces the risk of several other diseases, including metabolic diseases, which on the other hand are also associated with a lower microbial diversity, suggests that the context is of crucial importance when considering microbial diversity. For example, loss of microbiota diversity generally opens up niches for opportunistic invaders, ${ }^{41}$ whereas the plethora of bioactive components transferred by breastfeeding protect against colonization by such opportunistic pathogens. ${ }^{43}$ This further underscores the need for meticulous adjustment for diet as a confounding factor in the association between microbiota and disease outcomes.

Using various multivariable longitudinal analyses, we furthermore demonstrated that the microbial community structure, diversity, and maturity, as well as the relative abundance of several individual genera were associated with the subsequent development of allergic manifestation. We know from previous animal studies and large longitudinal human cohorts that intestinal microbial dysbiosis in allergic diseases is mainly observed within a critical window in early life. ${ }^{44}$ The comparability between studies is, however, hampered by the highly dynamic microbial communities within this early time window, which likely results in different associations at different sampling time points. The main strengths of the present study are its prospective design, repeated sample collection, and the deep clinical phenotyping.

The regular physical examinations of the children throughout the first 3 years of life in combination with the collection of detailed questionnaire data allowed not only deep clinical phenotyping, but also an accurate assessment of the time of disease onset. The follow-up into school age further facilitated a reliable classification of children who developed allergic asthma, as it is well known that wheezing symptoms at an earlier age are often transient and caused by episodic viral infections. ${ }^{45}$

We observed a lower microbial diversity to be associated with AD development and allergic sensitization, but not with asthma. This is consistent with previous studies that also reported a reduced microbial diversity in association with $\mathrm{AD}^{46-49}$ and sensitization. ${ }^{7,50}$ In contrast, a link between microbial diversity and wheeze or asthma often could not be observed. $^{51-53}$ Although atopic manifestations are common comorbidities, these results support previous conclusions 

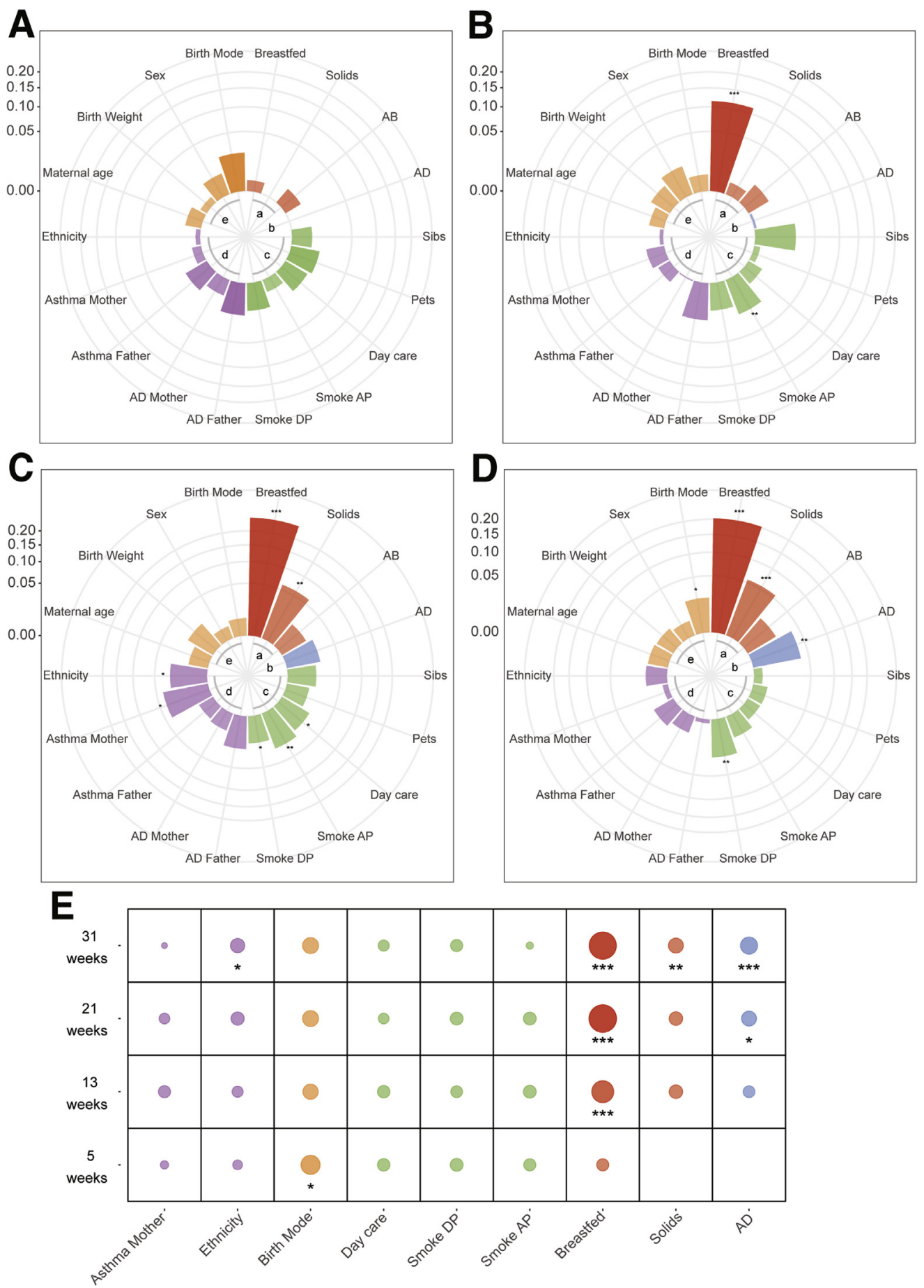

Figure 3. Microbiota community structure is most strongly influenced by breastfeeding $(\mathrm{n}=961$ stool samples from 312 children). ( $A-D)$ Polar plots visualizing the amount of variance of microbial communities at 5 weeks $(A), 13$ weeks $(B), 21$ weeks $(C)$, and 31 weeks $(D)$ of age that could be explained by 18 covariates as analyzed using EnvFit. The height of the bars reflects the amount of variance (r2) explained by each covariate. Covariates are colored to highlight parental health status and ethnicity (purple), perinatal covariates (orange), diet and medication (red), and environmental exposures (green). Asterisks indicate significant covariates (FDR $P<$ .05) at each time point. (E) Permutational multivariate analysis of variance combining all covariates that were significantly associated with microbial community variation at any given time point in the EnvFit analyses. The size of the dots reflects the $R^{2}$. Only samples without missing data on the included covariates were included in permutational multivariate analysis of variance ( 5 weeks: $\mathrm{n}=$ 238, 13 weeks: $n=233,21$ weeks: $\mathrm{n}=231,31$ weeks: $\mathrm{n}=259)$. Asterisks indicate statistical significance with ${ }^{\star} P<.05,{ }^{\star *} P<.01,{ }^{\star \star *} P<$ .001 . from the Copenhagen Prospective Study on Asthma in Childhood (COPSAC) that extrapolation of risk factors between different atopic disorders may not always be justified. $^{54}$

The increased risk of $\mathrm{AD}$, sensitization, and asthma among children with a higher microbial maturity might at first seem in contrast with the previously mentioned results for microbial diversity and with findings of previous studies. Indeed recent results from COPSAC $_{2010}$ linked a low microbial maturity with later onset of asthma in children born to asthmatic mothers. ${ }^{11}$ In our study, the microbial maturity was, however, only significantly increased at 5 weeks of age in children who developed sensitization (as determined by skin prick tests and serum IgE levels to the most common aeroallergens) and asthma. Also, for children with $\mathrm{AD}$, we observed a microbial maturity (MAZ) that was significantly higher at the earliest recorded time point but gradually decreased and became even lower at the age of 31 weeks when compared with the MAZ of children who did not develop AD. This temporally higher MAZ in very young 


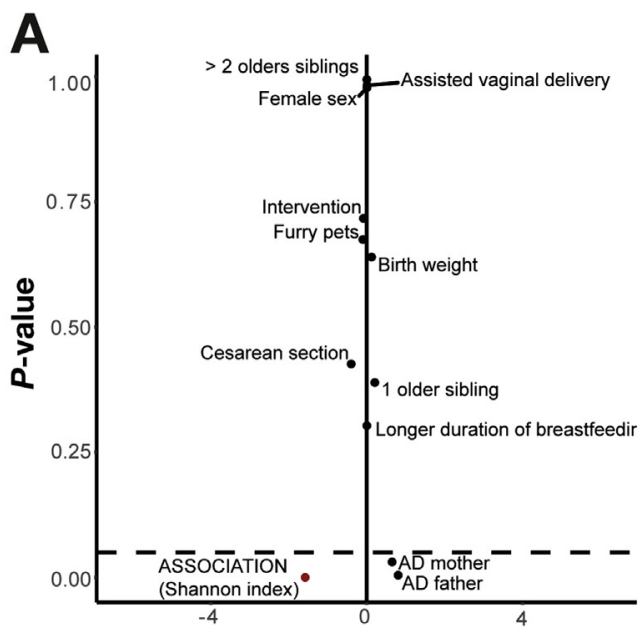

B
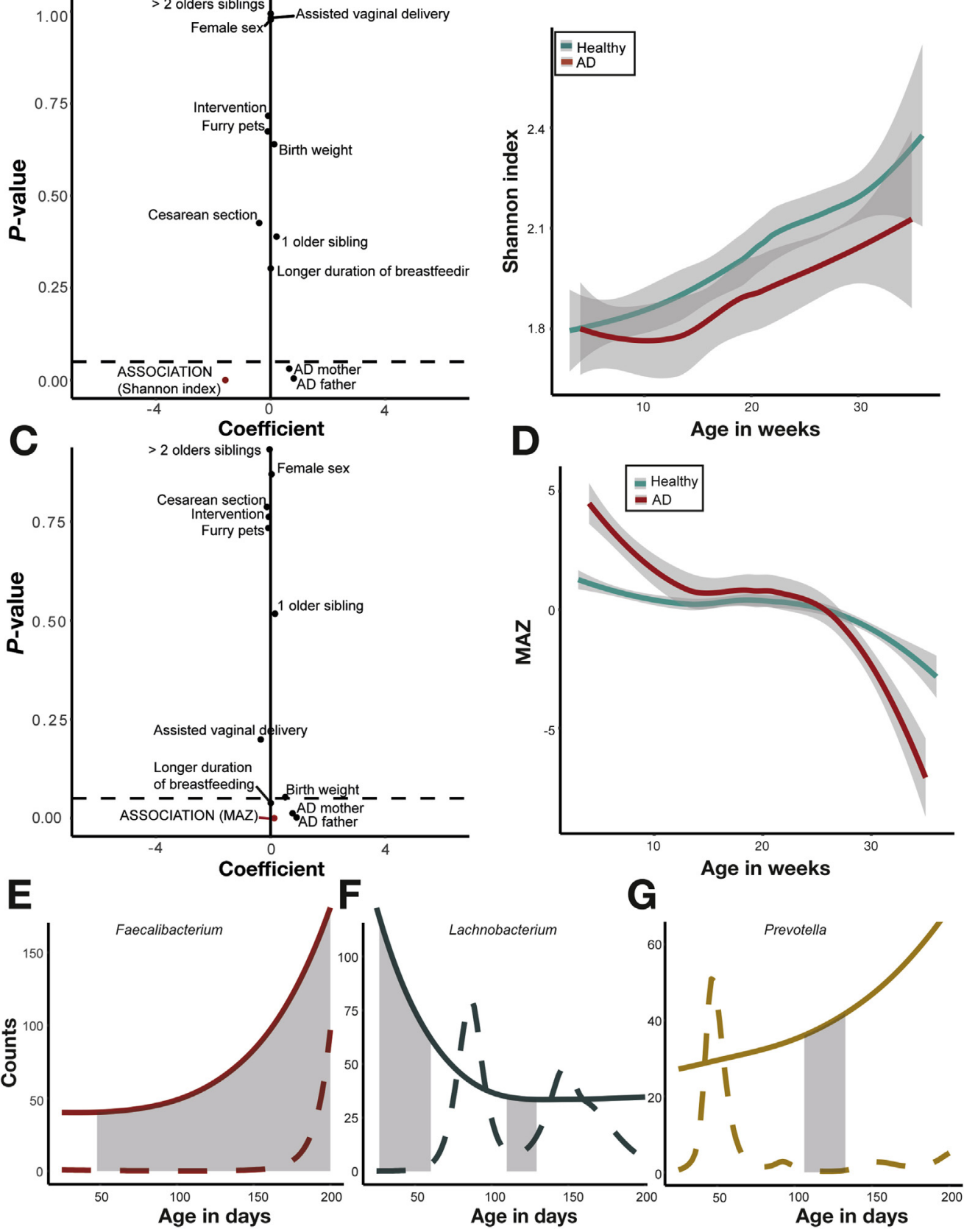

Figure 4. Microbiota composition, diversity, and maturity is linked to the subsequent development of $A D$ ( $n=961$ stool samples from 312 children). (A) Volcano plot depicting the regression coefficients from the joint model on the association between the Shannon index and AD. The dashed line depicts the threshold for statistical significance at $P<.05$. Variables depicted below the dashed line were statistically significantly associated with $A D$ in the final model. Positive coefficients (variables to the right of the vertical line) were associated with an increased AD risk. Negative coefficients (variables to the left of the vertical line) were associated with a decreased AD risk. The hazard ratio is given by the exponent of the coefficient (eg, for Shannon index: $e^{\wedge}-1.57$ results in a hazard ratio of 0.21$)$. (B) Development of microbial diversity (Shannon index) throughout infancy among children that did (red line) or did not (green line) develop AD, as modeled by Loess regression. Gray areas represent the $95 \%$ confidence intervals. (C) Volcano plot depicting the regression coefficients from the joint model on the association between the MAZ and the development of AD. The dashed line depicts the threshold for statistical significance at $P<.05$. $(D)$ Development of microbial maturity (MAZ score) throughout infancy among children who did (red line) or did not (green line) develop AD as modeled by Loess regression. Gray areas represent the 95\% confidence intervals. (E-G) Time intervals of differential abundance in Faecalibacterium $(E)$, Lachnobacterium $(F)$, and Prevotella $(G)$ between infants who did or did not develop $\mathrm{AD}$ as identified from MetaLonda analyses. Significantly different time intervals (FDR adjusted $P<.05)$ are depicted by gray shading. 
infants might therefore suggest a dysregulated colonization process (eg, with some bacterial taxa arriving [too] early), rather than a more mature overall microbial community structure.

Next to differences in microbial diversity and maturity, we were able to identify microbial taxa that were differentially abundant among infants who did or did not develop allergic disease manifestations. Lachnobacterium and Faecalibacterium were significantly decreased throughout infancy among children who developed AD. Also, Lachnospira and Dialister, next to Lachnobacterium, were significantly decreased among children who developed asthma.

The fact that these bacterial taxa were not only differentially abundant at a single time point but throughout infancy strengthens the likelihood of a causal role in the protection against allergic disease. Altogether, our results indicate that microbial perturbations in early life are also associated with asthma at school age, although perturbations are not identical to those observed in children who developed $\mathrm{AD}$. In line with our findings, analysis on the microbiota composition at 3 months of age within the Canadian Healthy Infant Longitudinal Development (CHILD) Study revealed Lachnospira and Faecalibacterium to be significantly decreased among children at risk for allergic wheeze at the age of 1 year. ${ }^{52}$ Moreover, a lower relative abundance of, among others, Lachnospiraceae incertae sedis, Faecalibacterium, and Dialister at the age of 1 year in children from COPSAC $_{2010}$ was associated with an increased risk of asthma at 5 years. $^{11}$

Fermentation products of these bacteria are a possible explanation for the protective effect of these bacteria. Acetate is one of the fermentation products of Lachnospira and to a lesser account Lachnobacterium. Animal studies have previously shown that acetate feeding leads to a marked suppression of allergic airway disease in a mouse-model for human asthma. The underlying cellular mechanism was related to the effect of acetate on Treg cells, particularly through epigenetic modification of the Foxp3 protomotor. $^{5}$

Faecalibacterium (prausnitzii) is well known for its antiinflammatory effects, among others, through the production of butyrate $^{55}$ and a microbial anti-inflammatory molecule that inhibits the nuclear factor- $\kappa$ B pathway. ${ }^{56}$ Two recent studies have identified another lactate-consuming butyrateproducing genus, Anaerostipes, associated with a decreased risk of food allergy ${ }^{57}$ and eczema. ${ }^{58}$ The very low abundance of this genus in our population could potentially explain the lack of association in our study.

The application of several types of longitudinal data analysis, including the joint modeling of longitudinal and survival data, which had previously not been used for microbiota data analyses, enabled us to demonstrate that alterations in microbial diversity, maturity, and composition preceded the clinical manifestations of atopic diseases. Although this statistical framework reveals the temporality of associations, thereby suggesting causal relationships, causality can never be proven in an observational study. For example, microbial perturbations could be an epiphenomenon of exposure to yet another unknown risk factor for allergy. Also, it cannot be ruled out that early preclinical manifestation of allergies or genetic predisposition for allergy might impact the microbiota composition. Moreover, our findings on fecal collections might not fully reflect alterations in the microbiome on allergy development at the level of (small) intestinal mucosa.

It is therefore of importance not only to replicate findings in similar cohorts, but also to conduct future experimental studies building on these findings to reveal the underlying biological mechanisms and prove causality.

In conclusion, our results demonstrate the importance of birth mode and diet on the early maturation of the infant microbiota and demonstrate that, on careful adjustment of important confounding factors, alterations in the microbial colonization process of the infant intestinal tract precede the development of $\mathrm{AD}$, sensitization, and asthma. In particular, members of the Lachnospiraceae family, as well as the genera Faecalibacterium and Dialister appear to protect against allergies. These findings further support the future development of evidence-based intervention strategies targeting the microbiota to prevent or treat allergic diseases in early life.

\section{Supplementary Material}

Note: To access the supplementary material accompanying this article, visit the online version of Gastroenterology at www.gastrojournal.org, and at https://doi.org/10.1053/ j.gastro.2020.01.024.

\section{References}

1. Bennek E, Mandic AD, Verdier J, et al. Subcellular antigen localization in commensal $E$. coli is critical for $T$ cell activation and induction of specific tolerance. Mucosal Immunol 2019;12:97-107.

2. Cahenzli J, Koller Y, Wyss M, et al. Intestinal microbial diversity during early-life colonization shapes long-term lgE levels. Cell Host Microbe 2013;14:559-570.

3. Stefka AT, Feehley T, Tripathi P, et al. Commensal bacteria protect against food allergen sensitization. Proc Natl Acad Sci U S A 2014;111:13145-13150.

4. Olszak T, An D, Zeissig S, et al. Microbial exposure during early life has persistent effects on natural killer $\mathrm{T}$ cell function. Science 2012;336:489-493.

5. Thorburn AN, McKenzie Cl, Shen S, et al. Evidence that asthma is a developmental origin disease influenced by maternal diet and bacterial metabolites. Nat Commun 2015;6:7320.

6. Trompette A, Gollwitzer ES, Yadava K, et al. Gut microbiota metabolism of dietary fiber influences allergic airway disease and hematopoiesis. Nat Med 2014;20:159-166.

7. Azad MB, Konya T, Guttman DS, et al. Infant gut microbiota and food sensitization: associations in the first year of life. Clin Exp Allergy 2015;45:632-643.

8. Dzidic M, Abrahamsson TR, Artacho A, et al. Aberrant $\lg A$ responses to the gut microbiota during infancy precede asthma and allergy development. J Allergy Clin Immunol 2017;139:1017-1025.e14.

9. Penders J, Stobberingh EE, van den Brandt PA, et al. The role of the intestinal microbiota in the 
development of atopic disorders. Allergy 2007; 62:1223-1236.

10. Simonyte Sjodin K, Hammarstrom ML, Ryden $\mathrm{P}$, et al. Temporal and long-term gut microbiota variation in allergic disease: a prospective study from infancy to school age. Allergy 2019;74:176-185.

11. Stokholm J, Blaser MJ, Thorsen J, et al. Maturation of the gut microbiome and risk of asthma in childhood. Nat Commun 2018;9:141.

12. Fujimura KE, Sitarik AR, Havstad S, et al. Neonatal gut microbiota associates with childhood multisensitized atopy and T cell differentiation. Nat Med 2016;22:1187-1191.

13. Zimmermann P, Messina N, Mohn WW, et al. Association between the intestinal microbiota and allergic sensitization, eczema, and asthma: a systematic review. J Allergy Clin Immunol 2019;143:467-485.

14. Simonyte Sjodin K, Vidman L, Ryden P, et al. Emerging evidence of the role of gut microbiota in the development of allergic diseases. Curr Opin Allergy Clin Immunol 2016;16:390-395.

15. Korpela K, Costea P, Coelho LP, et al. Selective maternal seeding and environment shape the human gut microbiome. Genome Res 2018;28:561-568.

16. Wampach L, Heintz-Buschart A, Fritz JV, et al. Birth mode is associated with earliest strain-conferred gut microbiome functions and immunostimulatory potential. Nat Commun 2018;9:5091.

17. Gilbert JA, Lynch SV. Community ecology as a framework for human microbiome research. Nat Med 2019; 25:884-889.

18. Davidson R, Roberts SE, Wotton CJ, et al. Influence of maternal and perinatal factors on subsequent hospitalisation for asthma in children: evidence from the Oxford record linkage study. BMC Pulm Med 2010;10:14.

19. van Nimwegen FA, Penders J, Stobberingh EE, et al. Mode and place of delivery, gastrointestinal microbiota, and their influence on asthma and atopy. J Allergy Clin Immunol 2011;128:948-955. e1-e3.

20. Lau S, Gerhold K, Zimmermann K, et al. Oral application of bacterial lysate in infancy decreases the risk of atopic dermatitis in children with 1 atopic parent in a randomized, placebo-controlled trial. J Allergy Clin Immunol 2012;129:1040-1047.

21. Penders J, Gerhold K, Stobberingh EE, et al. Establishment of the intestinal microbiota and its role for atopic dermatitis in early childhood. J Allergy Clin Immunol 2013;132:601-607.e8.

22. Bartram AK, Lynch MD, Stearns JC, et al. Generation of multimillion-sequence 16S rRNA gene libraries from complex microbial communities by assembling pairedend illumina reads. Appl Environ Microbiol 2011; $77: 3846-3852$.

23. Whelan FJ, Surette MG. A comprehensive evaluation of the sl1p pipeline for 16S rRNA gene sequencing analysis. Microbiome 2017;5:100.

24. Lagkouvardos I, Fischer S, Kumar N, et al. Rhea: a transparent and modular R pipeline for microbial profiling based on 16S rRNA gene amplicons. PeerJ 2017;5:e2836.

25. Oksanen J, Guillaume BF, Kindt R, et al. Vegan: Community Ecology Package. R Package Version. 2.5-3. CRAN, 2013.
26. Chen J. GUniFrac: Generalized UniFrac Distances. R Package Version. 1.1. CRAN, 2018.

27. Subramanian S, Huq S, Yatsunenko T, et al. Persistent gut microbiota immaturity in malnourished Bangladeshi children. Nature 2014;510:417-421.

28. Holmes I, Harris K, Quince C. Dirichlet multinomial mixtures: generative models for microbial metagenomics. PLoS One 2012;7:e30126.

29. Stewart CJ, Ajami NJ, O'Brien JL, et al. Temporal development of the gut microbiome in early childhood from the TEDDY study. Nature 2018;562:583-588.

30. Morgan XC, Tickle TL, Sokol H, et al. Dysfunction of the intestinal microbiome in inflammatory bowel disease and treatment. Genome Biol 2012;13:R79.

31. Cadarso Suarez C, Klein N, Kneib T, et al. Editorial "Joint modeling of longitudinal and time-to-event data and beyond". Biom J 2017;59:1101-1103.

32. Rizopoulos D. JM: an R package for the joint modelling of longitudinal and time-to-event data. J Stat Softw 2010;35:1-33.

33. Metwally AA, Yang J, Ascoli C, et al. MetaLonDA: a flexible $R$ package for identifying time intervals of differentially abundant features in metagenomic longitudinal studies. Microbiome 2018;6:32.

34. Azad MB, Konya T, Maughan H, et al. Gut microbiota of healthy Canadian infants: profiles by mode of delivery and infant diet at 4 months. CMAJ 2013;185:385-394.

35. Backhed F, Roswall J, Peng Y, et al. Dynamics and stabilization of the human gut microbiome during the first year of life. Cell Host Microbe 2015;17:690-703.

36. Penders J, Thijs C, Vink C, et al. Factors influencing the composition of the intestinal microbiota in early infancy. Pediatrics 2006;118:511-521.

37. Huang L, Chen Q, Zhao $Y$, et al. Is elective cesarean section associated with a higher risk of asthma? A metaanalysis. J Asthma 2015;52:16-25.

38. Thavagnanam S, Fleming J, Bromley A, et al. A metaanalysis of the association between Caesarean section and childhood asthma. Clin Exp Allergy 2008;38: 629-633.

39. Pannaraj PS, Li F, Cerini C, et al. Association between breast milk bacterial communities and establishment and development of the infant gut microbiome. JAMA Pediatr 2017;171:647-654.

40. Ho NT, Li F, Lee-Sarwar KA, et al. Meta-analysis of effects of exclusive breastfeeding on infant gut microbiota across populations. Nat Commun 2018;9:4169.

41. Bello MGD, Knight R, Gilbert JA, et al. Preserving microbial diversity. Science 2018;362:33-34.

42. Lin B, Dai R, Lu L, et al. Breastfeeding and atopic dermatitis risk: a systematic review and meta-analysis of prospective cohort studies [published online ahead of print November 6, 2019]. Dermatol doi: 10.1159/ 000503781.

43. van den Elsen LWJ, Garssen J, Burcelin R, et al. Shaping the Gut microbiota by breastfeeding: the gateway to allergy prevention? Front Pediatr 2019;7:47.

44. Stiemsma LT, Turvey SE. Asthma and the microbiome: defining the critical window in early life. Allergy Asthma Clin Immunol 2017;13:3. 
45. Townshend J, Hails S, McKean M. Diagnosis of asthma in children. BMJ 2007;335:198-202.

46. Abrahamsson TR, Jakobsson HE, Andersson AF, et al. Low diversity of the gut microbiota in infants with atopic eczema. J Allergy Clin Immunol 2012;129:434-440; 440. e1-e2.

47. Forno E, Onderdonk AB, McCracken J, et al. Diversity of the gut microbiota and eczema in early life. Clin Mol Allergy 2008;6:11.

48. Ismail IH, Oppedisano F, Joseph SJ, et al. Reduced gut microbial diversity in early life is associated with later development of eczema but not atopy in high-risk infants. Pediatr Allergy Immunol 2012;23:674-681.

49. Wang M, Karlsson C, Olsson C, et al. Reduced diversity in the early fecal microbiota of infants with atopic eczema. J Allergy Clin Immunol 2008;121:129-134.

50. Chen CC, Chen KJ, Kong MS, et al. Alterations in the gut microbiotas of children with food sensitization in early life. Pediatr Allergy Immunol 2016;27:254-262.

51. Arrieta MC, Arevalo A, Stiemsma L, et al. Associations between infant fungal and bacterial dysbiosis and childhood atopic wheeze in a nonindustrialized setting. J Allergy Clin Immunol 2018;142:424-434.e10.

52. Arrieta MC, Stiemsma LT, Dimitriu PA, et al. Early infancy microbial and metabolic alterations affect risk of childhood asthma. Sci Transl Med 2015;7:307ra152.

53. Bisgaard H, Li N, Bonnelykke K, et al. Reduced diversity of the intestinal microbiota during infancy is associated with increased risk of allergic disease at school age. J Allergy Clin Immunol 2011;128:646-652. e1-e5.

54. Bisgaard H, Halkjaer LB, Hinge R, et al. Risk analysis of early childhood eczema. J Allergy Clin Immunol 2009; 123:1355-1360.e5.

55. Canani RB, Costanzo MD, Leone L, et al. Potential beneficial effects of butyrate in intestinal and extraintestinal diseases. World J Gastroenterol 2011;17:1519-1528.

56. Quevrain E, Maubert MA, Michon C, et al. Identification of an anti-inflammatory protein from Faecalibacterium prausnitzii, a commensal bacterium deficient in Crohn's disease. Gut 2016;65:415-425.

57. Feehley $\mathbf{T}$, Plunkett $\mathbf{C H}, \mathbf{B a o} \mathbf{R}$, et al. Healthy infants harbor intestinal bacteria that protect against food allergy. Nat Med 2019;25:448-453.

58. Wopereis H, Sim K, Shaw A, et al. Intestinal microbiota in infants at high risk for allergy: effects of prebiotics and role in eczema development. J Allergy Clin Immunol 2018;141:1334-1342.e5.
Author names in bold designate shared co-first authorship

Received September 19, 2019. Accepted January 9, 2020.

Correspondence

Address correspondence to: John Penders, PhD, Department of Medical Microbiology, Maastricht University Medical Centre+, PO 5800, 6202 AZ,

Maastricht, The Netherlands. e-mail: j.penders@maastrichtuniversity.nl; fax: $+31-(0) 433876643$.

\section{Acknowledgments}

We thank Christel Driessen for lab analyses and Mayk Lucchesi for designing the graphical abstract.

Members of the GI-MDH consortium are listed as follows: Collaborators: Eileen K. Hutton, ${ }^{1}$ Katherine Morrison, ${ }^{2,3}$ Alison C. Holloway, ${ }^{1}$ Helen McDonald, ${ }^{4}$ Elyanne M. Ratcliffe, ${ }^{2,5}$ Jennifer C. Stearns, ${ }^{5,6}$ Jonathan D. Schertzer, ${ }^{3,5,7}$ Mike G. Surette, ${ }^{5,6,7}$ Lehana Thabane, ${ }^{8}$ Monique Mommers ${ }^{9,10}$

${ }^{1}$ Department of Obstetrics and Gynecology, McMaster University, Hamilton, Ontario, Canada. ${ }^{2}$ Department of Pediatrics, McMaster University, Hamilton, Ontario, Canada. ${ }^{3}$ Centre for Metabolism, Obesity, and Diabetes Research, McMaster University, Hamilton, Ontario, Canada.

${ }^{4}$ Midwifery Education Program, McMaster University, Hamilton, Ontario, Canada. ${ }^{5}$ Farncombe Family Digestive Health Research Institute, McMaster University, Hamilton, Ontario, Canada.

${ }^{6}$ Department of Medicine, McMaster University, Hamilton, Ontario, Canada. ${ }^{7}$ Department of Biochemistry \& Biomedical Sciences, McMaster University, Hamilton, Ontario, Canada.

${ }^{8}$ Department of Health Research Methods, Evidence, and Impact, McMaster University, Hamilton, Ontario, Canada. ${ }^{9}$ Department of Epidemiology, Care and Public Health Research Institute (CAPHRI), Maastricht University, Maastricht, The Netherlands. ${ }^{10}$ In Vivo Planetary Health: an affiliate of the World Universities Network (WUN), West New York, New Jersey.

\section{CRediT Authorship Contributions}

John Penders, PhD (Conceptualization: Supporting; Data curation: Supporting; Formal analysis: Supporting; Funding acquisition: Lead; Methodology: Lead; Supervision: Lead; Writing - original draft: Lead). Niels van Best, MSc (Conceptualization: Equal; Formal analysis: Equal; Methodology: Supporting; Writing - original draft: Equal; Writing - review \& editing: Equal). Gianluca Galazzo, MSc (Conceptualization: Supporting; Data curation: Lead; Formal analysis: Lead; Visualization: Lead; Writing - original draft: Equal). Mathias Hornef, Professor (Supervision: Equal; Writing - original draft: Supporting; Writing - review \& editing: Supporting). Paul Savelkoul, Professor (Conceptualization: Supporting; Supervision: Equal; Writing - original draft: Supporting; Writing - review \& editing: Supporting). Susanne Lau, Professor (Conceptualization: Lead; Funding acquisition: Lead; Investigation: Lead; Project administration: Lead; Writing - original draft: Supporting; Writing review \& editing: Supporting). Eckard Hamelmann, Professor (Conceptualization: Lead; Funding acquisition: Lead; Investigation: Equal; Writing - original draft: Supporting; Writing - review \& editing: Supporting). Liene Bervoets, PhD (Writing - original draft: Supporting; Writing - review \& editing: Supporting). Isaac Oteng Dapaah, MSc (Formal analysis: Equal; Writing - original draft: Supporting; Writing - review \& editing: Supporting).

\section{Conflict of interest}

The authors disclose no conflicts.

\section{Funding}

This study was financially supported by grants from the Joint Programming Initiative A healthy diet for a healthy life (HDHL) Joint Action Intestinal Microbiomics (project number 50-52905-98-599) and grant 2815ERA05E by the German Ministry of Nutrition and Agriculture (Bundesanstalt für Landwirtschaft und Ernährung) paid to Charité and Susanne Lau. The PAPS trial was funded by Symbiopharm Herborn and the German Research Foundation (DFG Ha 2162/4-1) paid to Eckard Hamelmann. 